\title{
CASTLEMAN DISEASE: A GREAT MIMICKER OF METASTASES IN RADIOIODINE REFRACTORY THYROID CANCER
}

\author{
Nazia Rashid ${ }^{1}$, Aamna Hassan ${ }^{1}$, Noreen Akhter ${ }^{2}$, Abdul Hameed ${ }^{3}$ \\ ${ }^{1}$ Department of Nuclear Medicine, Shaukat Khanum Memorial Cancer Hospital and Research Centre, Lahore, \\ ${ }^{2}$ Department of Pathology, Shaukat Khanum Memorial Cancer Hospital and Research Centre, Lahore, ${ }^{3}$ Department \\ of Haematology / Oncology, Ittefaq Hospital, Lahore, Pakistan
}

Received: 3 November 2018 / Accepted: 23 December 2018

\begin{abstract}
A 27-year-old male underwent total thyroidectomy for thyroid swelling. Histopathology showed papillary thyroid carcinoma $[\mathrm{T} 3-6.0 \mathrm{~cm}]$ with extra-thyroidal extension. The patient was treated with $150 \mathrm{mCi}$ radioactive iodine (RAI) as adjuvant ablative therapy. Radioiodine refractory disease was identified 1-year post-RAI therapy with elevated thyroglobulin levels and negative I-131 whole body scan. F-18 FDG positron emission tomography/computed tomography scan showed activity in the right thyroid bed and multilevel right cervical nodes. Right-sided modified neck dissection was done, which showed Castleman disease (hyaline vascular type) in right cervical nodes. The most probable cause of elevated tumour markers was found out to be $0.6 \mathrm{~cm}$ right thyroid bed nodule on follow-up ultrasonography. Our patient also had coexistent conditions as; osteopoikilosis and Hepatitis C along with thyroid carcinoma.
\end{abstract}

Key words: Castleman disease, lymph node, radioiodine, thyroid cancer

\section{Introduction}

Castleman disease (CD) first described in $1956^{[1]}$ is a rare lymphoproliferative disorder with diverse manifestations which can affect any organ. $\mathrm{CD}$ is a great mimicker of benign and malignant conditions. ${ }^{[2]}$ As in our patient, who was initially treated for papillary carcinoma thyroid. However, after the initial treatment with surgery and radioactive iodine therapy, patient developed radio-iodine refractory disease (RRD) resulting in no Iodine uptake on whole body scan although tumor markers were elevated. F-18 FDG PET-CT scan was performed to evaluate RRD and showed hypermetabolic lymph nodes. These were histologically proven to be involved with Castleman disease.

\section{Case Report}

A 27-year-old male presented with 3-year history of anterior neck swelling. Radionuclide Tc-99m thyroid scan showed a cold nodule. Fine-needle aspiration and cytology (FNAC)

Correspondence: Dr. Nazia Rashid, Department of Nuclear Medicine, Shaukat Khanum Memorial Cancer Hospital and Research Centre, Lahore, Pakistan.

Email:nazia5809@gmail.com suggested a follicular neoplasm Bethesda category-IV thyroid lesion. Hepatitis C (HCV) positivity was found incidentally on a pre-surgical screening test. The patient underwent total thyroidectomy which confirmed papillary thyroid carcinoma (PTC) $[$ T3 $-6.0 \mathrm{~cm}]$ with extrathyroidal extension. Baseline stimulated thyroglobulin (Tg) was $95 \mathrm{ng} / \mathrm{ml}$. Post-operative whole body scan (WBS) showed multifocal $\mathrm{I}^{131}$ avid thyroid remnant and patient was treated with $150 \mathrm{mCi}$ radioactive iodine (RAI) as adjuvant ablative therapy. Stimulated Tg levels 1-year post-therapy was $354 \mathrm{ng} / \mathrm{ml}$. However, I ${ }^{131}$ WBS did not reveal Iodine-avid disease indicating radioiodine-refractory disease (RRD). F-18 FDG positron emission tomography/computed tomography (PET/CT) scan showed subtle metabolic activity in the right thyroid bed with multilevel hypermetabolic right cervical nodes, suggesting active disease with dedifferentiation [Figure 1]. Right-sided modified neck dissection was done. Histopathology revealed Castleman disease (CD) (hyaline vascular type) in right cervical nodes with no evidence of malignancy or granulomatous disease. The patient remained on clinical follow-up. Ultrasound neck showed a $0.6 \mathrm{~cm}$ right thyroid bed nodule which was most likely the cause for elevated tumour markers. In addition, 
baseline and follow-up investigations (magnetic resonance imaging neck and PET/CT images) also showed incidental osteopoikilosis (OPK).

\section{Discussion}

\section{CD}

Historically in 1956, Dr. Benjamin Castleman described $\mathrm{CD}$ for the $1^{\text {st }}$ time. ${ }^{[1]} \mathrm{CD}$ is a rare lymphoproliferative disorder with diverse manifestations which may affect any organ. Therefore, it is a great mimicker of benign and malignant conditions. ${ }^{[2]}$ Only a few such cases are reported in literature. ${ }^{[3]} \mathrm{CD}$ is a non-cancerous, lymphoproliferative disorder which may have unicentric (UCD) or multicentric (MCD) presentation. ${ }^{[4]}$ MCD may exist as benign disease or pre-malignant disease which may transform into Hodgkin's lymphoma with poorer outcome. UCD disease is categorised as histologic evidence of CD in only one lymph node group with no clinical or radiographic evidence of lymphadenopathy elsewhere. On the other hand, MCD is categorised by histologic evidence of CD in one lymph node group with radiographic or clinical evidence of lymphadenopathy in other regions as well. ${ }^{[5]}$ In our patient, CD was found out in the right-sided levels II, III, and IV lymph nodes. Right-sided cervical nodal chain was the only side of FDG avidity on PET CT. Therefore, our patient was categorised under UCD.

Our patient is also a unique case where various independent entities coexist, i.e., thyroid cancer - PTC, OPK, HCV, and $\mathrm{CD}$. A review of literature shows no dependence or correlation between these entities except in between chronic/untreated HCV and CD. Chronic/untreated viral infection, i.e., HCV, EBV, and HHV-8 could initiate the systemic inflammatory response possibly leading to the development of CD. ${ }^{[5]}$ In our patient, the presence of HIV, EBV, or HHV-8 was not checked.

The diagnosis is made on histopathological features of excised affected lymph nodes. If excision is not possible, core needle biopsy is preferred over FNAC. ${ }^{[6]}$ Histopathological subtypes include hyaline vascular, plasma cell, mixed, and plasmoblastic subtypes. ${ }^{[7]}$ Histologically our patient shows hyalinevascular subtype which is characterised by an increased number of small, hyalinised blood vessels present within or in-between the follicles [Figure 2].

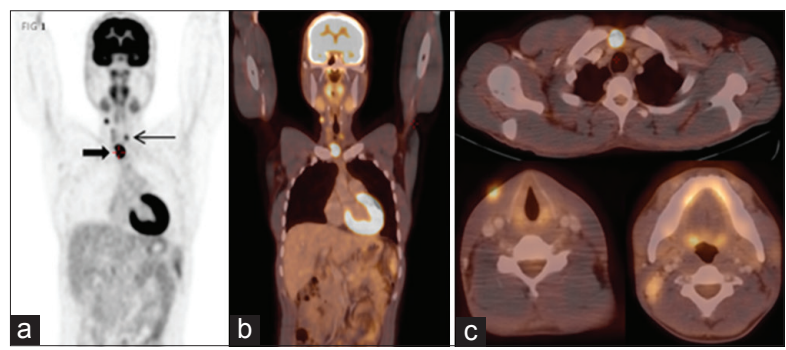

Figure 1: (a-c) F-18 FDG positron emission tomography/ computed tomography sagittal and coronal whole body fusion images showed hypermetabolic right level-II, III, and VI cervical lymph nodes with a standardised uptake value of 2.8 (thin arrow), these nodes represented Castleman disease. Focal uptake in the thyroidectomy bed with SUV of 4.8 (thick arrow) is residual thyroid disease

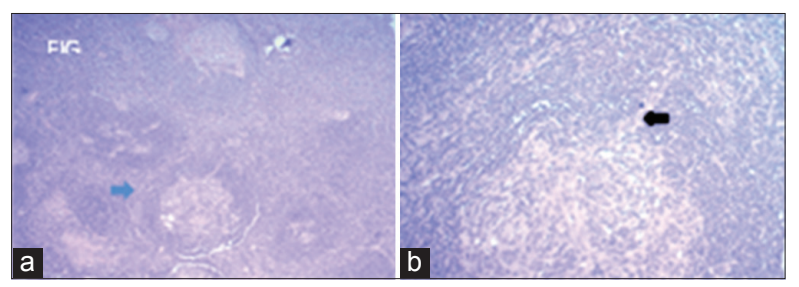

Figure 2: (a and b) Histopathology of our patient shows typical Onion skinning (blue arrow), which is formed by small lymphocytes of the mantle zones, arranged in concentric rings around the germinal centre and lollipop follicles (black arrow). Which are actually the follicles radially penetrated by blood vessels

There is an increase in lymphoid follicles with a predominance of dendritic cells within germinal centres as compared to lymphocytes. These features cause broadening of mantle zones. Two interesting features, which are part of the morphological spectrum are: (1) The onion skinning, (blue arrow) which is formed by small lymphocytes in the mantle zones arranged in concentric rings around the germinal centre and (2) lollipop follicles (black arrow), which are actually the follicles radially penetrated by blood vessels. Plasma cells are found in small clusters. The cases of plasma cell variant CD show greater retention of the nodal architecture with hyperplastic follicles of variable sizes and focally patent medullary sinuses. The inter-follicular region characteristically contains sheets of mature-appearing plasma cells. ${ }^{[8]}$

The disease mainly involves the mediastinum and abdomen. Peripheral involvement, especially of the 

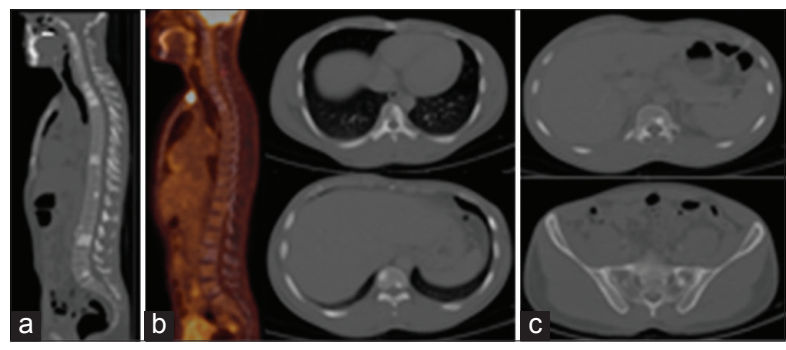

Figure 3: (a-c) Positron emission tomography/computed tomography (PET/CT): Fused sagittal and axial (CT component of PET/CT) images showing multilevel scattered sclerotic foci in the spine. No FDG uptake was seen at these levels. Follow-up imaging in 1 year showed a stable appearance. A diagnosis of atypical osteopoikilosis was made. Axial images show multilevel spotted bone disease

head and neck region, is rare. Differentials include lymphadenitis, tuberculosis, sarcoidosis, toxoplasmosis, cytomegalovirus, mononucleosis, HIV, and some tumours such as neurofibroma, cervical lipoma, Hodgkin's disease, thymoma, non-Hodgkin's lymphoma, and lymph nodal metastasis from head and neck malignancies..$^{[9]}$ The disease is staged before start of treatment to help in determining the centricity of disease, associated inflammatory manifestations, conditions, and malignancies. Staging helps in choosing appropriate treatment modalities. There are variable treatment options for UCD and MCD disease. Common sites of presentation in UCD include the chest (30\%), neck (23\%), abdomen (20\%), retroperitoneum $(17 \%)$ and rarely, the axilla $(5 \%)$, groin $(3 \%)$, or pelvis $(2 \%)$. Surgery is the established gold standard for the treatment of UCD.

Most UCD cases get a complete or partial response with surgery. While MCD cases respond to various chemotherapeutic agents, monoclonal antibodies, glucocorticoids, and thalidomide. Radiotherapy has also been reported to be effective in some patients with a variable response rate. ${ }^{[10-13]}$

\section{Papillary thyroid cancer}

PTC is the most common type of differentiated thyroid cancer with an excellent prognosis. A small percentage of PTC has the tendency to behave aggressively and develop into RRD. ${ }^{[14,15]}$ The modality of choice for imaging RRDis FDG PET-CT scan. ${ }^{[16]}$ Our patient represents an FDG avid case of $\mathrm{CD}$ unrelated to thyroid cancer. The elevated $\mathrm{Tg}$ levels were attributed to a sub centimetre recurrent right thyroid bed nodule. However, due to the small size of recurrent tissue, it could not be surgically resected.

\section{Osteopoikilosis}

OPK is rare benign condition manifesting as multifocal bone islands. It is usually incidentally found on radiographs or CT [Figure 3]; normal scintigraphic uptake on MDP bone scan strengthens the diagnosis by excluding bone metastasis. ${ }^{[17,18]}$ Although OPK is largely asymptomatic, $15-20 \%$ of patients present with slight articular pain and joint effusion. ${ }^{[19]}$ No definite pathogenesis is defined. However, multiple hypotheses have been suggested as hereditary failure to form normal trabeculae along the lines of stress, dysplasia of endochondral ossification, which can result in focal densities or striations within the trabecular bone, loss-of-function mutations in LEMD3 gene, and altered osteogenesis. ${ }^{[20]}$ First described in 1915 by AlbersSchonberg, ${ }^{[21]}$ OPK usually has multiple, sclerotic, round or oval foci with symmetrical distribution in periarticular areas within the epiphyseal and metaphyseal regions. However, slight variation is also noted in a few cases as in our patient.

In addition, thyroid cancer metastases are known to be lytic rather than dense sclerotic lesions. This feature, as well as lack of RAI and FDG PET/CT scan uptake in our patient, differentiated it from metastases.

\section{Conclusion}

$\mathrm{CD}$ is a potential mimicker of FDG avid metastatic disease on F18-FDG PET/CT scan in a variety of malignancies including undifferentiated thyroid cancer.

\section{Conflict of Interest}

The authors declare that they have no conflict of interest.

\section{References}

1. Castleman B, Iverson L, Menendez VP. Localized mediastinal lymphnode hyperplasia resembling thymoma. Cancer 1956;9:822-30.

2. Bonekamp D, Horton KM, Hruban RH, et al. Castleman disease: The great mimic. Radiographics 2011;31:1793-807.

3. Zeng YH, Chen CK, Lee CC. Castleman disease mimicking nodal recurrence of thyroid cancer. Endocrine 2016;51:384-6.

4. Lee HJ, Jeon HJ, Park SG, et al. Castleman's disease of the 
spleen. World J Gastroenterol 2015;21:1675-9.

5. Talukder DY, Delpachitra SN. Multicentric castleman's disease in a hepatitis C-positive intravenous drug user: A case report. Case Rep Med 2011;2011:567840.

6. Soumerai JD, Sohani AR, Abramson JS. Diagnosis and management of castleman disease. Cancer Control 2014;21:266-78.

7. Nagubandi R, Wang Y, Dutcher JP, et al. Classic case of unicentric mixed-type castleman's disease. J Clin Oncol 2013;31:e452-5.

8. Ferry J, Harris N. Atlas of Lymphoid Hyperplasia and Lymphoma. Philadelphia, PA: WB Saunders; 1997.

9. Amine $\mathrm{C}$, Abdelatif $\mathrm{O}$, Nouredine E. A rare case of castleman's disease presenting as cervical neck mass. Internet J Otorhinolaryngol 2008;9:1-4.

10. Talat N, Belgaumkar AP, Schulte KM. Surgery in castleman's disease: A systematic review of 404 published cases. Ann Surg 2012;255:677-84.

11. Dispenzieri A, Armitage JO, Loe MJ, et al. The clinical spectrum of castleman's disease. Am J Hematol 2012;87:997-1002.

12. Dispenzieri A, Gertz MA. Treatment of castleman's disease. Curr Treat Options Oncol 2005;6:255-66.

13. Bowne WB, Lewis JJ, Filippa DA, et al. The management of unicentric and multicentric castleman's disease: A report of 16 cases and a review of the literature. Cancer
1999;85:706-17.

14. Gild ML, Topliss DJ, Learoyd D, et al. Clinical guidance for radioiodine refractory differentiated thyroid cancer. Clin Endocrinol (Oxf) 2018;88:529-37.

15. Sfar R, Kamoun T, Nouira M, et al. Differentiated thyroid cancer with thyroglobulin elevation and negative iodine scintigraphy (TENIS syndrome). Int J Otolaryngol 2014;3:149-53.

16. Özdemir E, Yildirim Poyraz N, Polat SB, et al. Diagnostic value of $18 \mathrm{~F}-\mathrm{FDG}$ PET/CT in patients with TENIS syndrome: Correlation with thyroglobulin levels. Ann Nucl Med 2014;28:241-7.

17. Negi RS, Manchanda KL, Sanga S, et al. Osteopoikilosisspotted bone disease. Med J Armed Forces India 2013;69:196-8.

18. Tsai SY, Wang SY, Shiau YC, et al. Benign incidental findings of osteopoikilosis on tc-99m MDP bone SPECT/ CT: A case report and literature review. Medicine (Baltimore) 2016;95:e3868.

19. Korkmaz MF, Elli M, Özkan MB, et al. Osteopoikilosis: Report of a familial case and review of the literature. Rheumatol Int 2015;35:921-4.

20. Woyciechowsky TG, Monticielo MR, Keiserman B, et al. Osteopoikilosis: What does the rheumatologist must know about it? Clin Rheumatol 2012;31:745-8.

21. Di Primio G. Benign spotted bones: A diagnostic dilemma. CMAJ 2011;183:456-9. 